\title{
Atividade laboral dos pais de estu- dantes de segundo grau candidatos a Cursos de Graduação em Ciências da Saúde
}

\section{Professional activities of parents of High School students applicants to Health Sciences Courses}

Samuel R. Filipini ${ }^{1}$, Maristela M.S. Silva ${ }^{2}$, Marcia B. di Stasio ${ }^{3}$, Margarete de L. Ramos ${ }^{4}$, Marta N.C. Marçal-Vieira ${ }^{5}$, Cristiane M. Peres ${ }^{6}$, Maria de L.V. Rodrigues ${ }^{7}$

\begin{abstract}
RESUMO:
Modelo do estudo: Estudo baseado em coleta de informações por meio de Questionário Estruturado. Objetivo do estudo: Verificar indicadores da situação econômica e cultural das famílias de alunos do segundo grau que pleiteiam o ingresso em cursos destinados a formar profissionais da saúde.

Metodologia: Questionários auto-administrados foram respondidos por 256 estudantes do segundo grau, visitantes de Feira das Profissões da USP (FPUSP-interior). Esses questionários continham perguntas relativas ao tipo de escola que frequentavam e as atividades laborais de seus genitores. Os 108 estudantes de Escolas Públicas constituíram o Grupo 1 e os 148 alunos de Escolas Particulares formaram o Grupo 2.

Resultados: A atividade profissional paterna mais frequente no Grupo 1 foi a de "Técnico Manual"(42,15\%) e no Grupo 2 a de "Profissional de Nível Universitário" (24,11\%). O maior grupo de mães do Grupo 1 foi composto por "Donas de Casa" (22,33\%) seguido pelo das que exerciam "Outras Profissões Manuais"(21,36\%). No Grupo 2 a categoria mais frequente para as mães foi "Profissionais de Nível Universitário" (23,18\%) seguida por "Professores e Diretores de Escolas" (19,56\%). Cinco participantes do Grupo 1 e 27 do Grupo 2 eram filhos de Profissionais da Saúde.

Conclusões: Apesar das diferenças das atividades laborais predominantes em cada um dos grupos estudados, os resultados confirmam que aspiração de se tornar um Profissional da Saúde independe da formação ou das profissões dos pais.
\end{abstract}

Descritores: Estudantes. Profissões. Profissões em Saúde.

1) Técnico Acadêmico; 2) Técnico Administrativo da Comissão de Cultura e Extensão Universitária; 3) Auxiliar Administrativo do Centro de Apoio Educacional e Psicológico; 4) Técnico Acadêmico da Pró-Reitoria de Cultura e Extensão Universitária da USP; 5) Docente, Curso de Nutrição e Metabolismo (Departamento de Pediatria e Puericultura), Membro da CCEx, Coordenadora das atividades relacionadas à Feiras das Profissões, Membro do Grupo de Consultores do CAEP; 6) Educadora, Doutora, Membro da Equipe Técnica do CAEP; 7) Docente, Curso de Medicina (Departamento de Oftalmologia, Otorrinolaringologia e Cirurgia de Cabeça e Pescoço), Presidente da CCEx e Membro do Grupo de Consultores do CAEP. Faculdade de Medicina de Ribeirão Preto da Universidade de São Paulo (FMRP-USP).
Correspondência:

Maria de Lourdes Veronese Rodrigues Departamento de Oftalmologia, Otorrinolaringologia e Cirurgia de Cabeça e Pescoço, Faculdade de Medicina de Ribeirão Preto da Universidade de São Paulo. mdlvrodr@fmrp.usp.br

Artigo recebido em 07/01/2014 Aprovado para publicação em 13/05/2014 


\section{Introdução}

Aspecto de extrema importância na trajetória de todo ser humano é sua atividade laboral. Atualmente, nos deparamos com a diversidade profissional, a multiplicidade de competências necessárias para atender as demandas de uma sociedade complexa e como observa Sobrinho $(2005)^{1}$ sobre esse panorama, "não conseguimos compreender bem as transformações enquanto as vivemos, tão rapidamente os arranjos sociais se movem, e muito menos temos a menor certeza das possibilidades futuras". Portanto, mais difícil ficou para o jovem a tarefa da escolha profissional.

$\mathrm{O}$ assunto passou a encontrar lugar na Psicologia Vocacional que teve a expansão de suas práticas no contexto industrial do século XX. Com o decorrer dos anos acabou por espelhar a crescente complexidade dos fatores relevantes na escolha. Por conseguinte, nesse campo de investigação emergiram várias linhas teóricas assinalando a influência de fatores sócio-históricos, econômicos, culturais, cognitivos, familiares, dentre outros que levam o indivíduo a uma determinada profissão. Consequentemente a necessidade dos indivíduos serem mais bem orientados para essa escolha passou a ser também uma preocupação por parte do setor educacional.No sistema educacional brasileiro nas escolas privadas é comum encontrar serviços e atividades de orientação profissional pelos quais se realiza a análise de diferentes aspectos do mundo do trabalho e das áreas profissionais a fim de proporcionar uma reflexão mais aprofundada durante o processo de escolha de uma profissão. E o impacto da escola e de seus professores tem sido encontrado em um numero significativo de investigações. ${ }^{2,3}$

Contudo, no ensino secundário público, o processo de escolha profissional encontra poucos espaços de discussão junto aos estudantes. Ciente desse contexto, a Universidade de São Paulo se propôs, desde 2001, à divulgar os cursos oferecidos pelas suas Unidades junto aos estudantes de ensino médio. A universidade instituiu a Feira de Profissões da USP que é, a cada ano, organizada em um dos Campi do Interior e na capital do Estado de São Paulo. Segundo consta no site oficial do programa (http://prceu.usp.br/uspprofissoes), "o objetivo da itinerância é propiciar aos estudantes visitantes um conhecimento geral sobre a infraestrutura oferecida nos diferentes campi da Universidade de São Paulo, no interior." ${ }^{\text {S }}$ Sugerem que dessa forma, durante os três anos do ensino médio, os estudantes que ainda se encontram no seu primeiro ano terão oportunidade de conhecer in loco mais de um Campus e diversas das profissões oferecidas por eles. A feira é organizada no formato de estandes onde os estudantes recebem de professores alunos e/ou funcionários da USP esclarecimentos sobre os cursos, as especificidades das profissões e o processo de entrada na universidade (vestibular) ${ }^{4}$.

Nesse contexto, julgou-se importante identificar algumas das premissas teórico metodológica presentes em vários trabalhos que tratam do impacto da influencia da família em seus aspectos culturais na escolha profissional ${ }^{2,5}$.

Assim, os objetivos de presente estudo são verificar indicadores da situação econômica e cultural das famílias de alunos do segundo grau que pleiteiam o ingresso em cursos destinados a formar profissionais da saúde.

\section{Método}

Questionário auto-administrado foi aplicado a estudantes do segundo grau que visitaram Feira das Profissões da USP-Interior (FEPUSP-Interior) que continha, entre outras, referentes à motivações para a escolha da profissão, perguntas sobre o tipo de escola que frequentavam e sobre a profissão de seus pais.

Dos 265 visitantes que concordaram em participar do estudo (número correspondente a $6,15 \%$ dos 4.300 visitantes da FEPUSP-Interior estudada e a mais de metade dos que visitaram o estande da Faculdade de Medicina de Ribeirão Preto-USP e se declararam interessados em Ciências da Saúde), foram excluídos nove que não informaram a profissão de nenhum dos pais, sendo a população de estudo composta por 256 jovens (aproximadamente 5,9\% do total de visitantes e 66\% dos que se declararam interessados em Profissões da Saúde) - Figura 1. Cinquenta e um (19,92\%) dos participantes eram do sexo masculino e as idades do grupo total variaram entre 13 e 22 anos (mediana= 17 anos).

Estes estudantes foram divididos em dois grupos: Grupo A - alunos de escolas públicas ( $\mathrm{n}=108$; 20 homens) e Grupo-B - alunos de escolas particulares ( $\mathrm{n}=148 ; 31$ homens).

As profissões do pai e da mãe foram, inicialmente, codificadas com base na tabela do Ministério da Fazenda do Brasil. No entanto, foi necessário fazer adaptações e agrupamentos, para fins de análise.

Para verificar diferenças entre os grupos foram utilizados os testes do chi-quadrado ou Teste Exato de Fisher. 


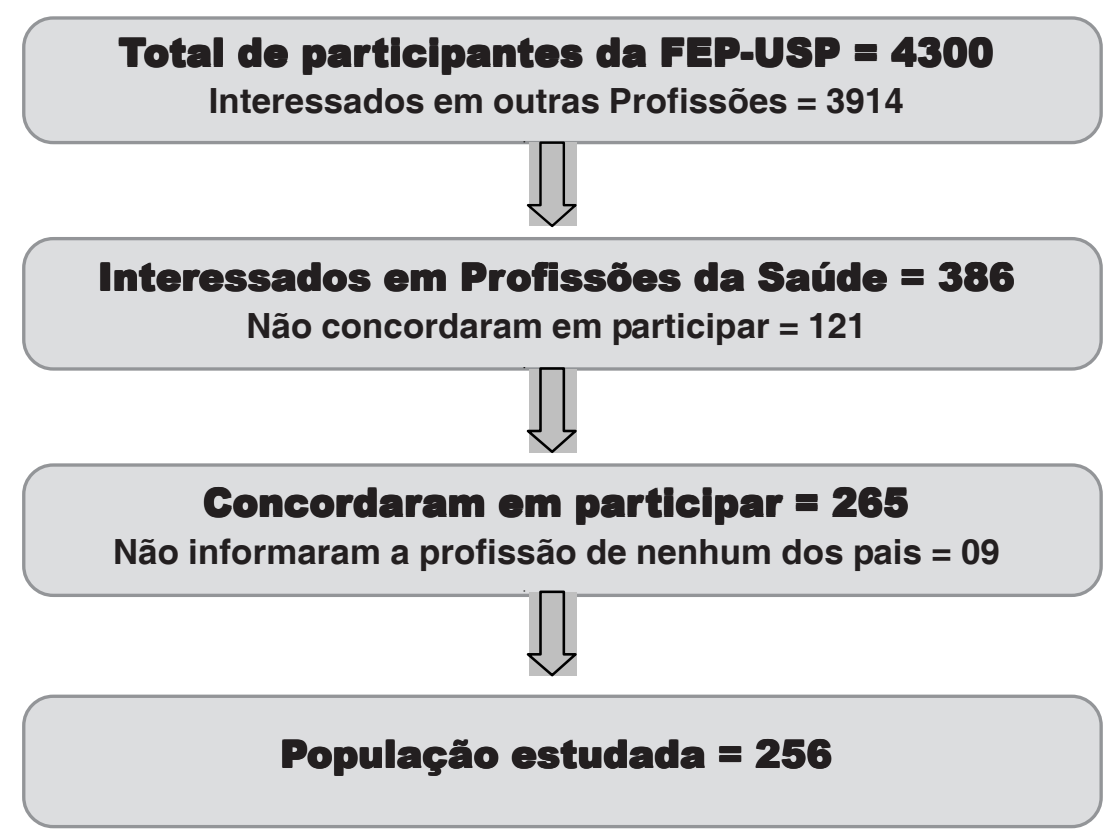

Figura 1: Representação gráfica da sequência de exclusões dos participantes da FEPUSP-Interior estudada.

\section{Resultados e Discussão}

A Feira recebeu em média cinco mil visitantes, entre estudantes, professores, pais e demais interessados, grande número dos quais visitaram o estande dos cursos da Faculdade de Medicina de Ribeirão Preto. Mas somente foram convidados a participar os que declararam intenção de prestar vestibular para um Curso de Ciências da Saúde. Dentre esses, duzentos e sessenta e cinco estudantes do segundo grau responderam ao questionário.

Vinte e oito $(10,93 \%)$ participantes só informaram a profissão de um dos pais, mas não foram excluídos por que este dado fornece indicativo da situação sócioeconômica da família. A profissão do pai deixou de ser informada por 13 estudantes (6 do G1 e 7 do G2) e a da mãe por quinze deles (cinco do grupo 1 e dez do grupo 2).

Na Tabela I está apresentada a distribuição dos pais e mães por agrupamento de categorias profissionais e por grupo de participantes, assim como as diferenças estatísticas entre os grupos.

No Grupo 1 o agrupamento profissional predominante $(42,15 \%)$ para os pais foi o de "Técnicos Manuais" (eletricistas, pedreiros, etc) e para as mães "Dona de Casa" (22,33), seguida de perto por "Outras Profissões Manuais" (21,36\%). As porcentagens de "Técnicos manuais" foram estatisticamente dife- rentes nos dois Grupos de pais $(\mathrm{p}<0.0001)$. Para as mães esta atividade só foi declarada por cinco estudantes do Grupo 1 e por um do Grupo 2. Para fins de análise, decidiu-se acrescentar "Outras Profissões Manuais", obtendo-se, também para as mães diferenças estatisticamente significantes $(\mathrm{p}<0,0001)$.

No Grupo $224,11 \%$ dos pais e $23,18 \%$ da mães eram profissionais de nível universitário, seguindo-se, em relação aos pais, os grupos "Empresários e Comerciantes" (17,02\%) e "Técnicos Manuais" (13,47\%). Depois de profissionais de nível universitário, o agrupamento predominante nas mães do Grupo 2 e foi o de "Professores e Diretores de Escola" (19,56\%).

Comparando a frequência de profissionais universitários nos dois grupos as diferenças foram estatisticamente significantes, como era esperado (para os pais $\mathrm{p}<0,0001$ e para as mães $\mathrm{p}=0,0003$ ). Seis participantes do Grupo 1 e 27 do Grupo 2 tinham pelo menos um dos pais trabalhando na Área da Saúde. Deste último grupo, dois estudantes eram filhos de casais de profissionais da saúde. As diferenças entre os grupos também foram significantes $(\mathrm{p}=0,0024)$.

Quanto aos "Professores e Diretores de Escolas", atividade com frequência importante nas mães do Grupo 2, houve diferenças estatística menor, quando comparados os dois grupos, uma vez que 7,76\% das mães do Grupo 1 também exerciam essa atividade $(\mathrm{p}=0,01)$. 
Tabela I: Distribuição de pais e mães que tiveram suas profissões informadas, por Grupo de estudantes e por Grupo Laboral.

\begin{tabular}{|c|c|c|c|c|c|c|c|c|}
\hline \multirow[b]{2}{*}{$\begin{array}{l}\text { Grupos de } \\
\text { Profissões } \\
\end{array}$} & \multicolumn{4}{|c|}{ Grupo 1} & \multicolumn{3}{|c|}{ Grupo 2} & \multirow[b]{2}{*}{ Valores de $p$} \\
\hline & $\begin{array}{r}N^{0} \text { de } \\
102 \\
\end{array}$ & $\begin{array}{l}\text { pais } \\
(\%)\end{array}$ & \multicolumn{2}{|c|}{$N^{0}$ de mães } & $\begin{array}{c}N^{\circ} \text { de } \\
141 \\
\end{array}$ & $\begin{array}{l}\text { pais } \\
(\%)\end{array}$ & $\begin{array}{l}N^{\circ} \text { de mães } \\
138(\%)\end{array}$ & \\
\hline $\begin{array}{l}\text { Profissionais de } \\
\text { nível universitário }\end{array}$ & & & & & & & & \\
\hline - Da Área da Saúde & 0 & & 6 & $(5,82)$ & 12 & $(8,51)$ & $15(10,87)$ & $<0,0001$ (pais) \\
\hline - Outras Áreas & 6 & $(5,88)$ & 0 & & & $(15,60)$ & $17(12,31)$ & 0,003 (mães) \\
\hline $\begin{array}{l}\text { Professores e } \\
\text { Diretores de Escola }\end{array}$ & 2 & $(1,96)$ & 8 & $(7,76)$ & 6 & $(4,25)$ & $27(19,56)$ & 0,01 \\
\hline $\begin{array}{l}\text { Bancários e } \\
\text { Funcionários Públicos }\end{array}$ & 4 & $(3,92)$ & 6 & $(5,82)$ & 12 & $(8,51)$ & $10 \quad(7,25)$ & ns \\
\hline $\begin{array}{l}\text { Empresarios e } \\
\text { Comerciantes }\end{array}$ & 7 & $(6,86)$ & 4 & $(3,88)$ & & $(17,02)$ & $16(11,59)$ & ns \\
\hline $\begin{array}{l}\text { Técnicos não } \\
\text { manuais }\end{array}$ & 4 & $(3,92)$ & 1 & $(0,097)$ & 4 & $(2,82)$ & $1 \quad(0,072)$ & $\mathrm{ns}$ \\
\hline Técnicos manuais & 43 & $(42,15)$ & 5 & $(4,85)$ & & $(13,47)$ & 0 & $<0,0001$ \\
\hline Outras manuais & 0 & & 22 & $(21,36)$ & 0 & & $3 \quad(2,17)$ & \\
\hline Agricultor & 1 & $(0,098)$ & 0 & & 3 & $(2,12)$ & 0 & $\mathrm{~ns}$ \\
\hline Agropecuarista & 0 & & 0 & & 2 & $(1,41)$ & 0 & $\mathrm{nr}$ \\
\hline $\begin{array}{l}\text { Vendedores e } \\
\text { Representantes } \\
\text { comerciais }\end{array}$ & 5 & $(4,90)$ & 2 & $(1,94)$ & 3 & $(2,12)$ & $3(2,17)$ & ns \\
\hline $\begin{array}{l}\text { Bombeiro, } \\
\text { Agente de Segurança, } \\
\text { Policial, Agente Penal }\end{array}$ & 5 & $(4,90)$ & 0 & & 3 & $(2,12)$ & 0 & ns \\
\hline Dona de Casa & 0 & & 23 & $(22,33)$ & 0 & & $23(16,66)$ & ns \\
\hline Outras não manuais & 12 & $(11,76)$ & 20 & $(19,41)$ & & $(10,63)$ & $16(11,59)$ & ns \\
\hline Autônomo & 7 & $(6,86)$ & 3 & $(2,91)$ & 11 & $(7,80)$ & $5 \quad(3,62)$ & ns \\
\hline Aposentado/Pensionista & 6 & $(5,88)$ & 3 & $(3,91)$ & 5 & $(3,54)$ & $2(1,44)$ & ns \\
\hline Total & 102 & & 103 & & 141 & & 138 & \\
\hline
\end{tabular}

$\mathrm{ns}=$ não significante $/ \mathrm{nr}=$ não realizado 
Atualmente, no Brasil o cenário de trabalho tem sido predominantemente feminino, e Nepomuceno e Witter $(2010)^{6}$ levam em consideração também que "as profissões ditas femininas são as que tendem a ter menores salários e menos prestígio". Sendo assim, chamou atenção o fato de que $22,33 \%$ das mães do Grupo 1 e 16,66\% das do Grupo 2 não exercerem atividades profissionais, dedicando-se ao cuidado do lar e da família.

Uma das falhas do presente estudo foi não solicitar informações sobre o grau de escolaridade dos pais, pois é sabido que, principalmente, professores, bancários e profissionais não manuais diversos frequentaram a universidade.

Ao contrário dos observado por Carvalho e Taveira (2009) $)^{2}$, os resultados desse trabalho indicaram que a aspiração de se tornar um Profissional da Saúde independeu dos pais serem profissionais dessa área. No entanto, podem haver outros fatores de influencia familiar, além da atividade laboral dos pais ${ }^{3}$. Deve ser ressaltado, também, que neste estudo não pesquisamos a influência de outros parentes.

Pretende-se então prosseguir com esta investigação, para verificar em nosso meio, se as atividades laborais e o perfil cultural das famílias de estudantes que já ingressaram em Cursos de Graduação das Áreas da Saúde é similar as dos aspirantes e se há fatores externos que predominem na escolha profissional dessas áreas.

\section{Agradecimentos}

Os autores agradecem ao Prof. Dr. José Fernando de Castro Figueiredo (in memorian), pela sua participação no desenho do estudo e ao médico Claudimar Amaro, pela colaboração na coleta dos dados.

\section{SUMMARY:}

Study design: Study based on collection of information by means of a Structured Questionnaire.

Objective of the study: To determine indicators of the economic and cultural situation of the families of high school students who intend to enroll in courses for the formation of health professionals.

Methodology: Self-report questionnaires were answered by 256 high school students who were visiting the Professions Fair of USP (FPUSP- inland). The questionnaires contained questions about the type of school the students frequented and the work activities of their parents. Group 1 consisted of 108 Public School students and Group 2 consisted of 148 Private School students.

Results: The most frequent paternal professional activity was "Manual Technician" (42.15\%) for Group 1 and "University Level Professional" (24.11\%) for Group 2 . The largest group of mothers in Group 1 consisted of "Housewives" (22.33\%), followed by the group of mothers who had "Other Manual Professions" $(21.36 \%)$. In Group 2, the most frequent category for the mothers was "University Level Professional" (23.18\%), followed by "Teachers and Directors of Schools" (19.56\%). Five participants in Group 1 and 27 in Group 2 were children of Health Professionals.

Conclusions: Despite the differences in the work activities predominating in each group studied, the results confirm the ambition to become a Health Professional regardless of the training or profession of the parents

Key words: Students. Occupations. Health Occupations.

\section{Referências}

1. Dias Sobrinho J. Dilemas da educação superior no mundo globalizado - sociedade do conhecimento ou economia do conhecimento. 1st. ed. São Paulo, Casa do Psicólogo, 2005.

2. Carvalho M, Taveira MC. Influência dos pais nas escolhas de carreira dos filhos: visão de diferentes atores. Rev bras orientac prof. 2009; 10:33-41.

3. Santos LMM. O papel da família e dos pares na escolhaprofissional. Psicol estud. 2005; 10:57-66.
4. Universidade de São Paulo - Pró-Reitoria de Cultura e Extensão Universitária. Programa a USP e as Profissões. http://prceu.usp.br/uspprofissoes/

5. Swanson JL, Fouad NL. Career Theory and Practice: Learning Throug Case Studies. 2ed. San Francisco, Sage. 2008.

6. Nepomuceno RF, Witter GP. Influência da família na decisão profissional: opinião de adolescentes. Psicol Esc Educ. 2010; 14:15-22. 